\title{
ON THE EXPLICIT INCORPORATION OF SURFACE EFFECTS INTO THE MULTIPHASE MIXTURE BALANCE LAWS
}

\author{
LINDA M. ABRIOLA \\ Department of Civil Engineering, University of Michigan, Ann Arbor, MI 48109-2125, U.S.A. \\ and \\ WILLIAM G. GRAY \\ Department of Civil Engineering, University of Notre Dame, Notre Dame, IN 46556, U.S.A.
}

(Received 5 March 1984; in revised form 16 May 1985)

\begin{abstract}
Interfacial conditions for multiphase flows are formulated on the microscale to include the possibility of surface effects. These conditions are then averaged to obtain macroscopic continuum equations which may be used in describing multiphase flows. A transformation is presented whereby the resultant macroscopic equations can be rewritten in a form identical to that which has been developed previously for a multiphase system not influenced by interfacial effects. This transformation is shown to have an impact on the forms of the intraphase stress tensor and heat flux vector in addition to the interphase stress and heat flux terms. Inclusion of interfacial effects is demonstrated to be essential to the proper understanding of phase interaction.
\end{abstract}

\section{INTRODUCTION}

Many past investigations have centered on the use of averaging theory to develop macroscopic balance equations for multiphase systems from the microscopic balance equations of continuum mechanics. A summary of these investigations is beyond the scope of this work. Those interested may refer to Bedford \& Drumheller (1983) for a comprehensive review of significant contributions in this area. In most of the previous averaging theories (including Delhaye, 1977; Nigmatulin, 1979; Hassanizadeh \& Gray, 1979a,b; and Ene, 1981) surface effects at interfaces were either explicitly or implicitly neglected. Surface properties (such as surface tension), however, are known to be important to many types of multiphase mixtures.

A few researchers have sought to include some of these surface effects in their derivations. Ishii (1975) used a time-averaging approach and employed a number of simplifying assumptions to develop a two-phase flow model which incorporated surface effects. A two-phase model was also developed by Drew (1971) and Drew \& Segel (1971) by averaging local equations twice in space and time. Although surface momentum effects were considered in their formulation, energy and entropy interfacial effects were not examined. Resultant macroscopic equations in the foregoing theories are often quite complex and specification of a number of additional constitutive relations are required to complete the system.

This paper will focus on the explicit incorporation of surface properties into the multiphase macroscopic balance equations by a volume-averaging approach. It will be demonstrated that, if various terms are redefined, the form of these equations need not be more complex than the form of those equations which have been developed with no regard for surface effects. Thus, previous work in multiphase equation development could be reinterpreted and applied to mixtures for which surface phenomena play an important role.

The following development builds upon the work set forth in Hassanizadeh \& Gray (1979a). In that paper, a general macroscopic balance equation was derived by averaging local balance equations over a representative elementary volume (REV). The macroscopic 
balance law for a property $\psi$ in a phase $\alpha$ is obtained as:

$$
\begin{aligned}
\frac{\partial}{\partial t}\left(\langle\rho\rangle_{\alpha} \bar{\psi}^{\alpha}\right)+\nabla \cdot\left(\langle\rho\rangle_{\alpha} \overline{\mathbf{v}}^{\alpha} \bar{\psi}^{\alpha}\right)-\nabla \cdot \mathbf{i}^{\alpha}-\langle\rho\rangle_{\alpha} \bar{f}^{\alpha} & \\
& -\langle\rho\rangle_{\alpha} e^{\alpha}(\rho \psi)-\langle\rho\rangle_{\alpha} \hat{I}^{\alpha}=\langle\rho\rangle_{\alpha} \bar{G}^{\alpha} .
\end{aligned}
$$

Here overbars and angle brackets are used to indicate mass weighted and phase volume averages, respectively. For convenience, notation has been summarized in the nomenclature section at the end of this paper.

Equation [1] is very general in that it accounts for the effects of mass exchange between the phases, net production of a property within a phase, and external supply of that property to a phase. This balance equation must be supplemented by an averaged conservation condition across the interfaces within a REV. Derivation of such a condition is the subject of the next section. Once this condition has been developed, the specific balance laws of mass, momentum, energy and entropy will be presented and examined to give new insight.

\section{FORMULATION OF THE AVERAGED JUMP CONDITION}

Local balance equations which govern mass, momentum, energy and entropy at an interface between two phases have been developed by a number of investigators. Scriven (1960) first examined the mass and momentum balances in moving surfaces for Newtonian fluids. His work was extended by Slattery $(1964,1967)$ to include the balance of energy and to account for exchange of mäss with the adjacent phases. General local interfacial balance equations were later developed by Delhaye (1974), Ishii (1975) and Moeckel (1975). These equations incorporated surface properties, surface sources and mass exchange effects. Moeckel's development is the most general and will be used here as the starting point for the derivation of a macroscopic relation.

This discussion will be restricted to interfaces of negligibly small mass, such that interface momentum and mechanical energy may be neglected. In addition, external supplies of a property to an interface will not be considered. The phases of the multiphase system are assumed to be microscopically nonpolar (i.e. no internal spin momenta and no body or surface couples act on a body). For such a system, the general local balance equation for a property $\psi$ at an $\alpha-\beta$ interface may be written as (Moeckel 1975):

$$
\left.\mathbf{n}^{\alpha \beta} \cdot[\rho(\mathbf{w}-\mathbf{v}) \psi+\mathbf{i}]\right|_{\alpha}+\left.\mathbf{n}^{\beta \alpha} \cdot[\rho(\mathbf{w}-\mathbf{v}) \psi+\mathbf{i}]\right|_{\beta}=\nabla^{\prime} \cdot \lambda_{s}^{\alpha \beta}+p_{s}^{\alpha \beta} .
$$

Here $\left.\right|_{\alpha}$ indicates that a term is to be evaluated in the limit as the interface is approached from the $\alpha$-phase side, $\lambda_{s}^{\alpha \beta}$ is a surface flux and $p_{s}^{\alpha \beta}$ is a surface production term. In this paper, the subscript $s$ means that the subscripted quantity pertains to an interfacial surface, a prime (') designates a variable as a surface vector (two dimensional) in the interface, and $\nabla^{\prime}$ denotes the two-dimensional surface gradient operator.

Equation [2] may be used to derive a constraint on the macroscopic balance equation [1]. The following development of this constraint has its roots in the work of Hassanizadeh (1979). In keeping with the averaging theory and criteria put forth in Hassanizadeh \& Gray (1979a), eq [2] will be averaged over all $\alpha-\beta$ interfaces within an REV $\left(\delta A_{\alpha \beta}\right)$ and then over the entire volume of the system $(V)$ to produce the following global balance equation:

$$
\begin{gathered}
\left.\int_{V} \frac{1}{\delta V} \int_{\delta A_{\alpha \alpha}} \rho \psi(\mathbf{w}-\mathbf{v}) \cdot \mathbf{n}^{\alpha \beta}\right|_{\alpha} \mathrm{d} a \mathrm{~d} V+\left.\int_{V} \frac{1}{\delta V} \int_{\delta A_{\alpha \beta}} \rho \psi(\mathbf{w}-\mathbf{v}) \cdot \mathbf{n}^{\beta \alpha}\right|_{\beta} \mathrm{d} a \mathrm{~d} V \\
+\left.\int_{V} \frac{1}{\delta V} \int_{\delta A_{\alpha \beta}} \mathbf{n}^{\alpha \beta} \cdot \mathbf{i}\right|_{\alpha} \mathrm{d} a \mathrm{~d} V+\left.\int_{V} \frac{1}{\delta V} \int_{\delta A_{\alpha \beta}} \mathbf{n}^{\beta \alpha} \cdot \mathbf{i}\right|_{\beta} \mathrm{d} a \mathrm{~d} V \\
=\int_{V} \frac{1}{\delta V} \int_{\delta A_{\alpha d}} \nabla^{\prime} \cdot \lambda_{s}^{\alpha \beta} \mathrm{d} a \mathrm{~d} V+\int_{V} \frac{1}{\delta V} \int_{\delta A_{\alpha S}} p_{s}^{\alpha \beta} \mathrm{d} a \mathrm{~d} V .
\end{gathered}
$$


Equation [3] may be manipulated to gain information about the exchange terms which appear in the general balance equation [1]. Summing [3] over all $\alpha$ and $\beta$ yields:

$2 \int_{V} \sum_{\alpha}\langle\rho\rangle_{\alpha}\left[e^{\alpha}(\rho \psi)+\hat{I}^{\alpha}\right] \mathrm{d} V=\int_{V} \sum_{\alpha} \sum_{\beta \neq \alpha} \frac{1}{\delta V} \int_{\delta A_{\alpha \beta}} \nabla^{\prime} \cdot \lambda_{s}^{\alpha \beta^{\beta}} \mathrm{d} a \mathrm{~d} V$

$$
+\int_{V} \sum_{\alpha} \sum_{\beta \neq \alpha} \frac{1}{\delta V} \int_{\delta A_{\alpha}} p_{s}^{\alpha \beta} \mathrm{d} a \mathrm{~d} V .
$$

A factor of two appears in the left-hand side of the abovementioned equation due to the double occurrence of integrals over the region $\delta A_{\alpha \beta}$ for each specific $\alpha-\beta$ combination. The form of equation [4] may be simplified by the application of Green's theorem in a surface to the first term on the right-hand side (see appendix A):

$$
\int_{V} \sum_{\alpha} \sum_{\beta \neq \alpha} \frac{1}{\delta V} \int_{\delta A_{\alpha \beta}} \nabla^{\prime} \cdot \lambda_{s}^{\alpha \beta^{\beta}} \mathrm{d} a \mathrm{~d} V=\int_{A} \sum_{\alpha} \frac{1}{\delta A} \int_{C_{a}} \nu_{\alpha \beta}^{\prime} \cdot \lambda_{s}^{\alpha \beta} \mathrm{d} l \mathrm{~d} A
$$

Here $C_{\alpha}$ is the curve formed by the intersection of all interfaces bordering the $\alpha$ phase ( $\Sigma_{\beta \neq \alpha} \delta A_{\alpha \beta}$ ) with $\delta A$, an infinitesimal element of $A$ and $\nu_{\alpha \beta}^{\prime}$ is a unit surface vector lying tangent to the $\alpha-\beta$ interface and normal to $C_{\alpha}$ (see figure 1). The integral $\int_{C_{a}} \nu_{\alpha \beta}^{\prime} \cdot \lambda_{s}^{\alpha \beta} \mathrm{d} l$ represents the flux of the property $\psi$ through all $\alpha$ interfaces across the surface $\delta A$.

A macroscopic flux density contributed by all interfaces in contact with the $\alpha$ phase may

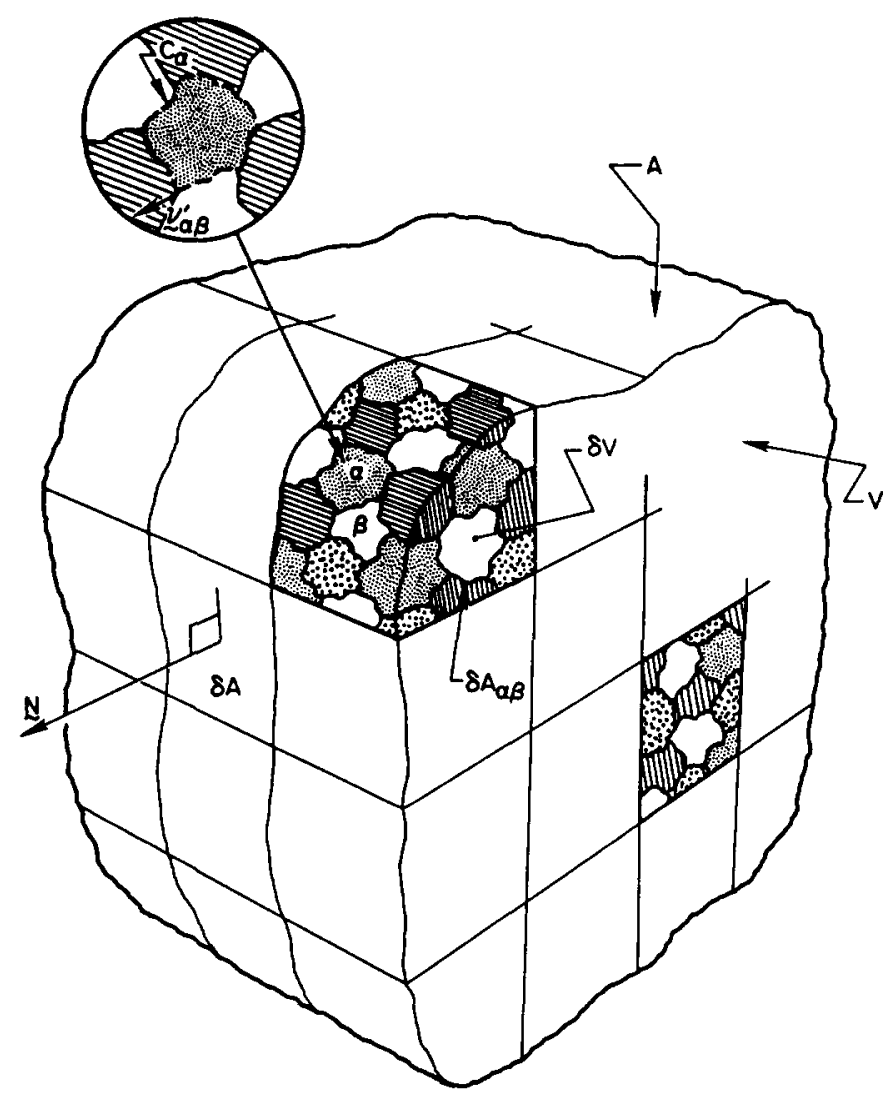

Figure 1. Schematic of multiphase system volume $V . V$ is subdivided into volumes $\delta V$ with total surface area $A$ subdivided into $\delta A$ and outward normal $N$. The interface between the $\alpha$ phase and all other phases is denoted by $\delta A_{\alpha \beta}$. The curve $C_{a}$ marks the intersection of $\delta A_{\alpha \beta}$ with $A$. The unit vector $\nu_{\alpha \beta}^{\prime}$ is normal to $C_{\alpha}$ and tangent to the $\alpha-\beta$ interface. 
now be defined as:

$$
\lambda_{s}^{\alpha}=\frac{1}{\delta A} \int_{C_{a}} \nu_{\alpha \beta}^{\prime} \cdot \lambda_{s}^{\alpha \beta} \mathrm{d} l
$$

Also definition of $\hat{p}_{s}^{\alpha}$ as

$$
\hat{p}_{s}^{\alpha}=\frac{1}{\delta V} \sum_{\beta \neq \alpha} \int_{\delta A_{\alpha j}} p_{s}^{\alpha \beta} \mathrm{d} a
$$

and substitution of [5] and [6] into [4] yields:

$$
2 \int_{V} \sum_{\alpha}\langle\rho\rangle_{\alpha}\left[e^{\alpha}(\rho \psi)+\hat{I}^{\alpha}\right] \mathrm{d} V=\int_{A} \sum_{\alpha} \lambda_{s}^{\alpha} \mathrm{d} A+\int_{V} \sum_{\alpha} \hat{p}_{s}^{\alpha} \mathrm{d} V .
$$

A tetrahedron argument (see Hassanizadeh \& Gray (1979a) appendix B) may be invoked at this point to prove the existence of a space flux vector $\lambda_{s}^{\alpha}$ such that $\mathbf{N} \cdot \lambda_{s}^{\alpha}=\lambda_{s}^{\alpha}$, where $\mathbf{N}$ is a unit normal to the surface $\delta A$. Application of the divergence theorem to the first term on the right-hand side of $[8]$ and localization of this equation produces the final form:

$$
2 \sum_{\alpha}\langle\rho\rangle_{\alpha}\left[e^{\alpha}(\rho \psi)+\hat{I}^{\alpha}\right]=\sum_{\alpha} \nabla \cdot \lambda_{s}^{\alpha}+\sum_{\alpha} \hat{p}_{s}^{\alpha}
$$

Equation [9] will serve as a constraint on the general balance equation [1].

\section{PRESENTATION OF THE BALANCE EQUATIONS}

Based on [1] and [9], individual macroscopic balance laws and their accompanying averaged interface conditions can now be developed. The balance laws have been derived previously by Hassanizadeh \& Gray (1979b) and will merely be stated here. Constraints of the specific form [9], however, do not seem to have appeared in the literature and hence will be presented in some detail.

The balance equations for a phase $\alpha$ may be written as:

Mass

$$
\frac{D^{\alpha}\langle\rho\rangle_{\alpha}}{D t}+\langle\rho\rangle_{\alpha}\left(\nabla \cdot \overline{\mathbf{v}}^{\alpha}\right)-\langle\rho\rangle_{\alpha} e^{\alpha}(\rho)=0
$$

\section{Linear Momentum}

$$
\langle\rho\rangle_{\alpha} \frac{D^{\alpha} \overline{\mathbf{v}}^{\alpha}}{D t}-\nabla \cdot \underline{t}^{\alpha}-\langle\rho\rangle_{\alpha} \overline{\mathbf{g}}^{\alpha}-\langle\rho\rangle_{\alpha}\left[\hat{\mathbf{T}}^{\alpha}+e^{\alpha}(\rho \tilde{\mathbf{v}})\right]=0 .
$$

Angular Momentum

$$
t^{\alpha}=\underline{t}^{\alpha^{r}}
$$

Energy

$$
\langle\rho\rangle_{\alpha} \frac{D^{\alpha} E^{\alpha}}{D t}-t^{\alpha}: \nabla \overline{\mathbf{v}}^{\alpha}-\nabla \cdot \mathbf{q}^{\alpha}-\langle\rho\rangle_{\alpha} h^{\alpha}-\langle\rho\rangle_{\alpha}\left[\hat{Q}^{\alpha}+e^{\alpha}(\rho \tilde{E})\right]=0
$$


Entropy

$$
\langle\rho\rangle_{\alpha} \frac{D^{\alpha} \bar{S}^{\alpha}}{D t}-\nabla \cdot \phi^{\alpha}-\langle\rho\rangle_{\alpha} \bar{b}^{\alpha}-\langle\rho\rangle_{\alpha}\left[\hat{\Phi}^{\alpha}+e^{\alpha}(\rho \tilde{S})\right]=\langle\rho\rangle_{\alpha} \bar{\Gamma}^{\alpha}
$$

Definitions of terms which appear in [10] are enumerated in the nomenclature section of this paper.

An itemization of the local quantities which must be substituted into [2] to obtain the individual interface equations is given in table 1. For the mass balance, both $p_{s}^{\alpha \beta}$ and $\lambda_{s}^{\alpha \beta}$ are zero since the interface has been assumed massless. In addition, there is no nonadvective flux of mass. Thus, the averaged mass jump condition [9] becomes:

$$
\sum_{\alpha}\langle\rho\rangle_{\alpha} e^{\alpha}(\rho)=0
$$

In the linear momentum balance at the interface, $\sigma_{s}^{\alpha \beta^{*}}$ represents a surface stress tensor which accounts for the effect of stress within the interface as well as surface tension. In indicial notation, $\sigma_{s}^{\alpha \beta}$ is the tensor $\left(\sigma_{s}^{\alpha \beta}\right)^{k_{\mu}}$ where $k$ is a spatial coordinate index varying from 1 to 3 while $\mu$ is a surface coordinate index varying from 1 to 2 . This convention has been used also by Moeckel (1975). Since there is no surface production of momentum, the averaged momentum jump condition takes the form:

$$
\sum_{\alpha}\langle\rho\rangle_{\alpha}\left[e^{\alpha}(\rho \tilde{\mathrm{v}})+e^{\alpha}(\rho) \overline{\mathrm{v}} \overline{\mathrm{w}}^{\alpha}+\hat{\mathrm{T}}^{\alpha}\right]=\frac{1}{2} \sum_{\alpha} \nabla \cdot \sigma_{s}^{\alpha}
$$

where

$$
\mathrm{N} \cdot \underline{\sigma}_{s}^{\alpha} \equiv \sigma_{s}^{\alpha} \equiv \frac{1}{\delta A} \int_{C_{\alpha}} \nu_{\alpha \beta}^{\prime} \cdot \sigma_{s}^{\alpha \beta} \mathrm{d} l
$$

Note $g_{s}^{\alpha}$ is a macroscopic spatial stress tensor which arises due to local stresses at all interfaces which contact the $\alpha$-phase.

Manipulation of the angular momentum interface condition along with [12] (see appendix B) will show that the sum of the macroscopic interfacial stress tensors is symmetric. That is,

$$
\sum_{\alpha} \sigma_{s}^{\alpha}=\left(\sum_{\alpha} \sigma_{s}^{\alpha}\right)^{T}
$$

\begin{tabular}{|c|c|c|c|c|}
\hline & $\psi$ & $\mathbf{i}$ & $\lambda_{s}^{\alpha \beta}$ & $p_{s}^{\infty \phi}$ \\
\hline Mass & 1 & 0 & 0 & 0 \\
\hline Linear momentum & v & t & $\sigma_{s}^{\text {afs }}$ & 0 \\
\hline Angular momentum & $r \times v$ & $r \times t$ & $\mathbf{r} \times \bar{x}_{s}^{\alpha f r}$ & 0 \\
\hline Energy & $E+\frac{1}{2} v^{2}$ & $t \cdot v+q$ & $g_{s}^{\alpha f} \cdot w+k_{s}^{\alpha f}$ & 0 \\
\hline Entropy & $s^{2}$ & $\phi$ & $\eta_{s}^{\alpha s}$ & $\Gamma$ \\
\hline
\end{tabular}

although $\sigma_{s}^{\alpha}$ for each $\alpha$ has not been demonstrated to be symmetric. It should be observed that this result holds only for microscopically nonpolar materials.

In the averaging of the energy interfacial condition, it is convenient to define the

Table 1. Definition of local quantities 
following quantity:

$$
\hat{k}_{s}^{\alpha} \equiv \hat{\mathbf{k}}_{s}^{\alpha} \cdot \mathbf{N} \equiv \frac{1}{\delta A} \int_{C_{\alpha}} \nu_{\alpha \beta}^{\prime} \cdot\left(\sigma_{s}^{\alpha \beta^{\prime}} \cdot \hat{\mathbf{w}}+\mathbf{k}_{s}^{\alpha \beta}\right) \mathrm{d} l .
$$

Here $\hat{w}$ is a space vector defined as the difference between the interface and mass average velocities:

$$
\hat{\mathbf{w}}=\mathbf{w}-\overline{\mathbf{v}}^{\alpha} \text {. }
$$

$\hat{\mathbf{k}}_{s}^{\alpha}$ as defined above represents the heat flux due to intrainterfacial heat transfer along with the microscopic work performed by the interfacial stress. One possible physical interpretation of $\hat{\mathbf{k}}_{s}^{\alpha}$ is that it accounts for temperature discontinuities between adjacent phases. The averaged energy jump condition can now be written as:

$$
\begin{aligned}
\sum_{\alpha}\langle\rho\rangle_{\alpha}\left[e^{\alpha}(\rho) E^{\alpha}+e^{\alpha}(\rho \tilde{E})+e^{\alpha}(\rho \tilde{\mathbf{v}})\right. & \cdot \overline{\mathbf{v}}^{\alpha}+\frac{1}{2} \bar{v}^{\alpha^{2}} e^{\alpha}(\rho) \\
& \left.+\hat{\mathbf{T}}^{\alpha} \cdot \overline{\mathbf{v}}^{\alpha}+\hat{Q}^{\alpha}\right]=\frac{1}{2} \sum_{\alpha} \nabla \cdot\left[\underline{\sigma}_{s}^{\alpha} \cdot \overline{\mathbf{v}}^{\alpha}+\hat{\mathbf{k}}_{s}^{\alpha}\right]
\end{aligned}
$$

The first term on the right-hand side of this equation represents the change in energy due to the macroscopic work performed by the interfacial stress.

Finally, the entropy interface condition may be given by:

$$
\sum_{\alpha}\langle\rho\rangle_{\alpha}\left[\hat{\Phi}^{\alpha}+e^{\alpha}(\rho \tilde{S})+e^{\alpha}(\rho) S^{\alpha}\right] \geq \frac{1}{2} \sum_{\alpha} \nabla \cdot \eta_{s}^{\alpha}
$$

Here $\eta_{s}^{\alpha} \equiv \mathbf{N} \cdot \eta_{s}^{\alpha}$ is a surface entropy flux. Note that the entropy production term has been omitted from [15]. Because this term is always non-negative (by the second law of thermodynamics), the balance condition is written as an inequality.

Much previous work in mixture theory (e.g. Bowen 1982; Passman et al. 1983) has made use of various forms of interfacial constraints. Rather than derive these constraints by the use of averaging principles, however, investigators have chosen to postulate their form. Such postulated constraint forms differ from those derived above in that their right hand sides are zero. In the next portion of this work, it will be shown that such a form can be consistent with an averaging theory approach if parameters are reinterpreted. Such reinterpretation, however, must necessarily be accounted for in any constitutive theory.

\section{EXAMINATION OF THE ENTROPY INEQUALITY}

The balance equations [10] along with the interfacial constraints [11-15] completely describe a multiphase system for which there is no macroscopic surface of discontinuity. Because they are no longer needed for clarification, all overbars and angle brackets will be dropped in the remaining portion of this paper. All variables which appear in subsequent equations are understood to represent macroscopic quantities. The remainder of this work will proceed under the common assumption that the entropy flux and external entropy supply terms are similarly proportional to the heat flux and external heat supply respectively such that

$$
\phi^{\alpha}=\frac{1}{\theta^{\alpha}} q^{\alpha} \quad \text { and } \quad b^{\alpha}=\frac{1}{\theta^{\alpha}} h^{\alpha}
$$


The parameter of proportionality, $\theta^{\alpha}$, is called the temperature of the $\alpha$ phase.

Consider now the entropy balance equation [10e]. Equations [16] may be substituted into this equation and $h^{\alpha}$ eliminated by the use of the energy balance equation [10d] to achieve:

$$
\begin{aligned}
\rho^{\alpha} \epsilon_{\alpha} \Gamma^{\alpha} & =\rho^{\alpha} \epsilon_{\alpha} \frac{D^{\alpha} S^{\alpha}}{D t}-\left(\frac{q_{k}^{\alpha}}{\theta^{\alpha}}\right)_{, k} \\
& -\frac{1}{\theta^{\alpha}}\left\{\rho^{\alpha} \epsilon_{\alpha} \frac{D^{\alpha} E^{\alpha}}{D t}-t_{k l}^{\alpha} v_{l, k}^{\alpha}-q_{k, k}^{\alpha}-\rho^{\alpha} \epsilon_{\alpha}\left[\hat{Q}^{\alpha}+e^{\alpha}(\rho \tilde{E})\right]\right\}-\rho^{\alpha} \epsilon_{\alpha}\left[\hat{\Phi}^{\alpha}+e^{\alpha}(\rho \tilde{S})\right] .
\end{aligned}
$$

Here indicial notation has been employed (summation convention implied for indices $k$ and $l)$ and $\langle\rho\rangle_{\alpha}$ has been replaced by $\rho^{\alpha} \epsilon_{\alpha}$, where $\rho^{\alpha}$ is the intrinsic mass density of the $\alpha$-phase and $\epsilon_{\alpha}$ is the void fraction of the $\alpha$-phase. If Helmholtz's free energy, $A^{\alpha}$, is defined as:

$$
A^{\alpha}=E^{\alpha}-\theta^{\alpha} S^{\alpha}
$$

then [17a] may be rewritten as:

$$
\begin{aligned}
\rho^{\alpha} \epsilon_{\alpha} \Gamma^{\alpha}=\frac{1}{\theta^{\alpha}}\left\{-\rho^{\alpha} \epsilon_{\alpha}[\right. & \left.S^{\alpha} \frac{D^{\alpha} \theta^{\alpha}}{D t}+\frac{D^{\alpha} A^{\alpha}}{D t}\right]= \\
& \left.+t_{k l}^{\alpha} \nu_{l, k}^{\alpha}+\frac{q_{k}^{\alpha}}{\theta^{\alpha}} \theta_{, k}^{\alpha} \overline{-} \rho^{\alpha} \epsilon_{\alpha}\left[\hat{Q}^{\alpha}+e^{\alpha}(\rho \tilde{E})-\theta^{\alpha}\left[\hat{\Phi}^{\alpha}+e^{\alpha}(\rho \tilde{S})\right]\right]\right\}
\end{aligned}
$$

Recalling the second law of thermodynamics which states that the net entropy production rate of a system must be non-negative, [17b] may be summed over all $\alpha$ phases to yield:

$$
\begin{aligned}
\sum_{\alpha} \frac{1}{\theta^{\alpha}}\left\{-\rho^{\alpha} \epsilon_{\alpha}\left[S^{\alpha} \frac{D^{\alpha} \theta^{\alpha}}{D t}+\frac{D^{\alpha} A^{\alpha}}{D t}\right]\right. & \left.+t_{k l}^{\alpha} v_{l, k}^{\alpha}+\frac{q_{k}^{\alpha}}{\theta^{\alpha}} \theta_{, k}^{\alpha}\right\} \\
& +\sum_{\alpha} \frac{\rho^{\alpha} \epsilon_{\alpha}}{\theta^{\alpha}}\left\{\left[\hat{Q}^{\alpha}+e^{\alpha}(\rho \tilde{E})\right]-\theta^{\alpha}\left[\hat{\Phi}^{\alpha}+e^{\alpha}(\rho \tilde{S})\right]\right\} \geq 0 .
\end{aligned}
$$

The entropy equation [18] can now be expanded by incorporating the interfacial constraints derived in the preceeding section. For this expansion, it will be convenient to select one phase, $r$, as a "reference phase." Thus, temperatures and velocities of other phases may be expressed with respect to those parameters for the reference phase. Let

$$
\begin{aligned}
& \theta^{\alpha r}=\theta^{\alpha}-\theta^{r} \\
& \mathbf{v}^{\alpha r}=\mathbf{v}^{\alpha}-\mathbf{v}^{r},
\end{aligned}
$$

then the operator $D^{\alpha} / D t$ may be expanded as:

$$
\frac{D^{\alpha}}{D t}(\cdot)=\frac{D^{\prime}}{D t}(\cdot)+v_{k}^{\alpha r}(\cdot)_{, k}
$$

Incorporation of [11-15] along with definitions [19], [20] into [18] results, after a great deal 
of algebraic manipulation, in the following form:

$$
\begin{aligned}
& \sum_{\alpha} \frac{1}{\theta^{\alpha}}\left\{-\rho^{\alpha} \epsilon_{\alpha}\left[\frac{D^{r} A^{\alpha}}{D t}+S^{\alpha} \frac{D^{r} \theta^{\alpha}}{D t}+v_{k}^{\alpha r} A_{, k}^{\alpha}+v_{k}^{\alpha r} S^{\alpha} \theta_{, k}^{\alpha}\right]\right. \\
& \left.+v_{l, k}^{\alpha}\left[t_{k l}^{\alpha}+\frac{1}{2} \sigma_{s_{k l}}^{\alpha}\right]+\frac{1}{\theta^{\alpha}}\left[q_{k}^{\alpha}+\frac{1}{2} k_{s_{k}}^{\alpha}\right] \theta_{, k}^{\alpha}\right\} \\
& -\sum_{\alpha} \frac{\rho^{\alpha} \epsilon_{\alpha}}{\theta^{\alpha}} A^{\alpha} e^{\alpha}(\rho)-\sum_{\alpha} \frac{\theta^{\alpha r}}{\theta^{\alpha} \theta^{r}}\left\{\rho^{\alpha} \epsilon_{\alpha}\left[\hat{Q}^{\alpha}+e^{\alpha}(\rho \tilde{E})+E^{\alpha} e^{\alpha}(\rho)\right]\right. \\
& \left.-\frac{1}{2} k_{s_{k, k}}^{\alpha}-\frac{1}{2} \sigma_{k l}^{\alpha} v_{l, k}^{\alpha}\right\}-\frac{1}{\theta^{r}} \sum_{\alpha}\left\{\rho^{\alpha} \epsilon_{\alpha}\left[\frac{1}{2} v_{k}^{\alpha r} v_{k}^{\alpha r} e^{s}(\rho)+v_{k}^{\alpha r} e^{\alpha}\left(\rho \tilde{v}_{k}\right)\right]\right. \\
& \left.\quad-v_{k}^{\alpha r}\left[\frac{1}{2} \sigma_{s_{k l, s}}^{\alpha}-\rho^{\alpha} \epsilon_{\alpha} \hat{T}_{k}^{\alpha}\right]\right\}+\frac{1}{2} \sum_{\alpha}\left\{\left(\frac{k_{s_{k}}^{\alpha}}{\theta^{\alpha}}-\eta_{s_{k}}^{\alpha}\right)_{, k}\right] \geq 0 .
\end{aligned}
$$

The last term in [21] brings to mind the constitutive equations [16]. If it is assumed that the interfacial heat flux term is similarly proportional to the interfacial entropy flux, i.e.:

$$
\eta_{s_{k}}^{\alpha}=\frac{k_{s_{k}}^{\alpha}}{\theta^{\alpha}}
$$

then the last term in [21] will vanish. A less restrictive constitutive assumption, but one producing the same result might be:

$$
\sum_{\alpha} \eta_{s_{k}}^{\alpha}=\sum_{\alpha} \frac{k_{s_{k}}^{\alpha}}{\theta^{\alpha}}
$$

A close inspection of [21] will reveal that the interfacial terms always appear in conjunction with certain of the mixture parameters. The following definitions are proposed:

$$
\begin{gathered}
\overline{\bar{q}}_{k}^{\alpha}=q_{k}^{\alpha}+1 / 2 k_{s_{k}}^{\alpha}, \\
\overline{\bar{t}}_{k l}^{\alpha}=t_{k l}^{\alpha}+1 / 2 \sigma_{s_{k l},}^{\alpha}, \\
\rho^{\alpha} \epsilon_{\alpha} \overline{\bar{T}}_{k}^{\alpha}=\rho^{\alpha} \epsilon_{\alpha} \hat{T}_{k}^{\alpha}-1 / 2 \sigma_{s_{k, j},}^{\alpha}, \\
\rho^{\alpha} \epsilon_{\alpha} \overline{\bar{Q}}^{\alpha}=\rho^{\alpha} \epsilon_{\alpha} \hat{Q}^{\alpha}-1 / 2 k_{s_{k, k}}^{\alpha}-1 / 2 \sigma_{s_{k l}}^{\alpha} v_{l, k}^{\alpha} .
\end{gathered}
$$

Here $\overline{\bar{q}}_{k}^{\alpha}$ represents some total heat flux due to both mixture and surface effects. Similarly, $\overline{\bar{t}}_{k l}^{\alpha}$ is some total stress tensor. The term $\rho^{\alpha} \epsilon_{\alpha} \overline{\bar{T}}_{k}^{\alpha}$ accounts for the exchange of momentum due to both mechanical interactions and surface tension. The exchange of energy due to mechancial interactions and interfacial effects is represented by $\rho^{\alpha} \epsilon_{\alpha} \overline{\bar{Q}}^{\alpha}$.

Incorporation of definitions [23] and [22a] into [21] yields a final form of the entropy inequality:

$$
\begin{aligned}
\sum_{\alpha} & \frac{1}{\theta^{\alpha}}\left\{-\rho^{\alpha} \epsilon_{\alpha}\left[\frac{D^{r} A^{\alpha}}{D t}+S^{\alpha} \frac{D^{r} \theta^{\alpha}}{D t}+v_{k}^{\alpha r} A_{, k}^{\alpha}+v_{k}^{\alpha r} S^{\alpha} \theta_{, k}^{\alpha}\right]\right. \\
& \left.+\overline{\bar{t}}_{k l}^{\alpha} v_{l, k}^{\alpha}+\frac{\overline{\bar{q}} \alpha k}{\theta^{\alpha}} \theta_{, k}^{\alpha}\right\}-\sum_{\alpha} \frac{\rho^{\alpha} \epsilon_{\alpha}}{\theta^{\alpha}} A^{\alpha} e^{\alpha}(\rho) \\
& -\sum_{\alpha} \frac{\theta^{\alpha r}}{\theta^{\alpha} \theta^{r}} \rho^{\alpha} \epsilon_{\alpha}\left\{\overline{\bar{Q}}^{\alpha}+e^{\alpha}(\rho \tilde{E})+E^{\alpha} e^{\alpha}(\rho)\right\} \\
& -\frac{1}{\theta^{r}} \sum_{\alpha}\left[\rho^{\alpha} \epsilon_{\alpha}\left\{\frac{1}{2} v_{k}^{\alpha r} v_{k}^{\alpha r} e^{\alpha}(\rho)+v_{k}^{\alpha r} e^{\alpha}\left(\rho \tilde{v}_{k}\right)+v_{k}^{\alpha \alpha} \overline{\bar{T}}_{k}^{\alpha}\right\}\right] \geq 0 .
\end{aligned}
$$


If this equation is compared to that developed for a multiphase system without surface effects (Gray 1983), the two equations are found to be identical in form though different in the way that terms must be interpreted.

In view of the definitions [23], the equations [10] and constraints [11-15] may also be rewritten as:

Mass

$$
\frac{\partial}{\partial t}\left(\rho^{\alpha} \epsilon_{\alpha}\right)+\left(\rho^{\alpha} \epsilon_{\alpha} v_{k}^{\alpha}\right)_{, k}-\rho^{\alpha} \epsilon_{\alpha} e^{\alpha}(\rho)=0
$$

Linear Momentum

$$
\rho^{\alpha} \epsilon_{\alpha} \frac{D^{\alpha} v_{l}^{\alpha}}{D t}-\overline{\bar{t}}_{k l, k}^{\alpha}-\rho^{\alpha} \epsilon_{\alpha} g_{l}^{\alpha}-\rho^{\alpha} \epsilon_{\alpha} \overline{\bar{T}}_{l}^{\alpha}-\rho^{\alpha} \epsilon_{\alpha} e^{\alpha}\left(\rho \tilde{v}_{l}\right)=0 .
$$

Angular Momentum

$$
\sum_{\alpha} \overline{\bar{t}}_{k l}^{\alpha}=\sum_{\alpha} \overline{\bar{t}}_{l k}^{\alpha}
$$

Energy

$$
\rho^{\alpha} \epsilon_{\alpha} \frac{D^{\alpha} E^{\alpha}}{D t}-\overline{\bar{t}}_{k l}^{\alpha} v_{l, k}^{\alpha}-\overline{\bar{q}}_{k, k}^{\alpha}-\rho^{\alpha} \epsilon_{\alpha} h^{\alpha}-\rho^{\alpha} \epsilon_{\alpha} \overline{\bar{Q}}^{\alpha}-\rho^{\alpha} \epsilon_{\alpha} e^{\alpha}(\rho \tilde{E})=0
$$

subject to:

$$
\begin{gathered}
\sum_{\alpha} \rho^{\alpha} \epsilon_{\alpha} e^{\alpha}(\rho)=0 \\
\sum_{\alpha} \rho^{\alpha} \epsilon_{\alpha}\left(e^{\alpha}(\rho) v_{l}^{\alpha}+e^{\alpha}\left(\rho \tilde{v}_{l}\right)+\overline{\bar{T}}_{l}^{\alpha}\right)=0 \\
\sum_{\alpha} \rho^{\alpha} \epsilon_{\alpha}\left(\frac{1}{2} v_{l}^{\alpha} v_{l}^{\alpha} e^{\alpha}(\rho)+v_{l}^{\alpha} e^{\alpha}\left(\rho \tilde{v}_{l}\right)+e^{\alpha}(\rho) E^{\alpha}+e^{\alpha}(\rho \tilde{E})+v_{l}^{\alpha} \overline{\bar{T}}_{l}^{\alpha}+\overline{\bar{Q}}^{\alpha}\right)=0 .
\end{gathered}
$$

Here explicit incorporation of interfacial properties into the balance equations has led to the interesting result that the macroscopic fluid stress tensor, $\overline{\bar{t}}^{\alpha}$, defined in [23b] is not necessarily symmetric. As stated by [25c], only the sum of these tensors has been shown to be symmetric for mixtures in which surface tension is of importance. With the exception of [25c], however, the system of equations [25] and [26] is found to be identical in form to those equations and constraints presented by Hassanizadeh \& Gray (1979b).

Thus, it has been demonstrated that there exists a transformation, (definitions [23]) of the flux terms for the macroscopic multiphase balance equations whereby these equations can maintain the same structure as those balance equations which govern a system not influenced by interfacial effects. The system [25] and [26] is entirely consistent with that postulated by Bowen (1982) for multiphase mixtures. It should be pointed out that although the form of the equations is unchanged by the inclusion of surface effects, the additional effects must be accounted for in the constitutive theory.

\section{DISCUSSION}

The importance of incorporating surface effects in the mixture balance laws can be illustrated by the following simple example. Consider a three-phase air $(g)$, water $(w)$, and 
solid $(r)$ system which is in capillary equilibrium (i.e. there is no movement of fluids nor mass exchange between phases). For simplicity, suppose that the medium is laterally homogeneous such that the saturation profile is essentially one dimensional and that soil porosity does not vary in space. For this system, the linear momentum constraint [12] becomes:

$$
\sum_{\alpha}\langle\rho\rangle_{\alpha} \hat{\mathbf{T}}^{\alpha}=\sum_{\alpha} \frac{1}{\delta V} \sum_{\beta \neq \alpha} \int_{\delta A_{\alpha S}} \mathbf{n}^{\alpha \beta} \cdot \underline{t} \mathrm{~d} a=\frac{1}{2} \sum_{\alpha} \nabla \cdot \sigma_{s}^{\alpha} .
$$

It can be shown by the use of various averaging theorems (e.g. Gray 1975) that:

$$
-\frac{1}{\delta V} \sum_{\beta \neq \alpha} \int_{\delta A_{\alpha \beta}} \mathbf{n}^{\alpha \beta} \mathrm{d} a=\nabla \epsilon_{\alpha}
$$

Since there is no flow in the system, $t$ is an equilibrium stress which varies linearly in space. By use of this fact and identity [28], constraint [27] may be rewritten as:

$$
-\left.\sum_{\alpha} \nabla \epsilon_{\alpha} \cdot \underline{t}^{\alpha}\right|_{e}=\frac{1}{2} \sum_{\alpha} \nabla \cdot q_{s}^{\alpha}
$$

where the subscript $e$ refers to some equilibrium value. Equation [29a] may be expanded as:

$$
-\left.\nabla \epsilon_{g} \cdot \underline{t}^{g}\right|_{e}+\left.\nabla \epsilon_{g} \cdot t^{w}\right|_{e}=\frac{1}{2} \sum_{\alpha} \nabla \cdot \underline{\sigma}_{s}^{\alpha} .
$$

Here, use has been made of the fact that $\nabla \epsilon_{r}=0$ for the system under consideration and that $\Sigma_{\alpha} \epsilon_{\alpha}=1$ by the definition of void fraction. Let the equilibrium stress be replaced by the relation:

$$
\left.\mathfrak{t}^{\alpha}\right|_{\varepsilon}=-P_{\alpha} I
$$

where $P_{\alpha}$ is the pressure of the $\alpha$ phase. Incorporation of [30] into [29b] then yields:

$$
-\left(P_{w}-P_{g}\right) \nabla \epsilon_{g}=\frac{1}{2} \sum_{\alpha} \nabla \cdot g_{s}^{\alpha}
$$

For the system under consideration, the vertical saturation profile will not be uniform $\left(\nabla \epsilon_{g} \neq 0\right)$. If the right-hand side of [31] were set to zero (as, for example, it was implicity in the work of Hassanizadeh \& Gray $(1979 \mathrm{a}, \mathrm{b})$ ), this would imply that $P_{w}=P_{g}$ (i.e. that the capillary pressure between the two fluids is zero). Only by incorporation of surface tension effects can one obtain the correct result that $P_{w} \neq P_{g}$.

Recall that, if one chooses to use constraints of the form [26], surface effects can still be incorporated into the mixture theory if certain parameters are redefined as in equations [28]. One must then be cautious, however, in the development of a constitutive theory for the system.

The principle of phase separation (Drew \& Segel 1971) is commonly applied in mixture theory to reduce the general dependency of constitutive relations. This principle is the assumption that the internal state of one phase is unrelated to the internal state of another. Examination of the expressions [23a, b] for fluid stress and heat flux, reveals that these variables will have an interphase dependence due to the surface terms $\boldsymbol{g}_{s}^{\alpha}$ and $\mathbf{k}_{s}^{\alpha}$. Thus, it should be recognized that the inclusion of surface effects is not compatible with the principle of phase separation for these variables. 


\section{CONCLUSIONS}

In this paper, surface properties have been explicitly incorporated into a system of macroscopic balance laws for a mixture by the averaging of local interface conditions. A transformation has been presented whereby this system can be rewritten in a form identical to that which has been described previously in the literature for a multiphase system not influenced by interfacial effects. By this transformation, the effective stress tensor and heat flux vector for each phase are defined and shown to depend on interfacial properties. Phase interaction terms $\overline{\bar{T}}_{k}^{\alpha}$ and $\overline{\bar{Q}}^{\alpha}$ are also shown to exhibit an interfacial dependence.

The importance of the inclusion of surface effects for the description of multiphase mixtures was illustrated by a simple example for an unsaturated porous medium in equilibrium. It was demonstrated that, for this system, one cannot account for capillary pressure without incorporation of these surface terms.

Acknowledgement-This work was supported in part by grant number DE-AC0283ER60170 from the Department of Energy and by grant number CEE79-20996 from the National Science Foundation.

\section{NOTATION}

\section{Special Notation/Definitions}

$\frac{D^{\alpha}}{D t}(\psi)=\frac{\partial}{\partial t}(\psi)+\overline{\mathbf{v}}^{\alpha} \cdot \nabla(\psi) \quad$ material derivative

$e^{\alpha}(\rho \psi)=\frac{1}{\langle\rho\rangle_{\alpha} \delta V} \sum_{\beta \neq \alpha} \int_{\delta A_{\alpha \beta}} \rho \psi(\mathbf{w}-\mathbf{v}) \cdot \mathbf{n}^{\alpha \beta} \mathrm{d} a \quad \begin{aligned} & \text { density function for the exchange } \\ & \text { of } \psi \text { due to phase change }\end{aligned}$

$\hat{I}^{\alpha}=\frac{1}{\langle\rho\rangle_{\alpha} \delta V} \sum_{\beta \neq \alpha} \int_{\delta A_{\alpha}} \mathbf{n}^{\alpha \beta} \cdot \mathbf{i} \mathrm{d} a \quad \begin{aligned} & \text { production of property } \psi \text { due to } \\ & \text { mechanical interaction }\end{aligned}$

$\langle\psi\rangle_{\alpha}(\mathbf{x}, t)=\frac{1}{\delta V} \int_{\delta V} \psi(\mathbf{x}+\xi, t) \gamma_{\alpha}(\mathbf{x}+\xi, t) \mathrm{d} V \quad$ phase volume average

$\bar{\psi}^{\alpha}(\mathbf{x}, t)=\langle\rho \psi\rangle_{\alpha} /\langle\rho\rangle_{\alpha} \quad$ mass average

$=$ term is modified to account for surface effects

$\nabla^{\prime}$ two-dimensional surface gradient operator $\left[\nabla^{\prime} \cdot \mathbf{f}^{\prime}=\left(f^{\mu}\right),_{\mu}\right.$ where $\mu$ is a surface coordinate index]

$\left[\nabla^{\prime} \cdot \underline{f}^{\prime}=\left(f^{k \mu}\right)_{\mu}\right.$ where $\mu$ is a surface coordinate index and $k$ is a spatial coordinate index.]

$\nabla \quad$ spatial gradient operator

$\sum_{\alpha}$ summation over all phases

- denotes surface (two-dimensional) vector

$\sum_{\beta \neq \alpha}$ summation over all $N$ phases except the $\alpha$ th

$\tilde{\psi}^{\alpha}=\psi-\bar{\psi}^{\alpha}$ deviation term

l. equilibrium value

\section{Roman letters}

$A$ surface area of $V$

$A^{\alpha} \quad$ Helmholtz free energy

$b$ external supply of entropy 


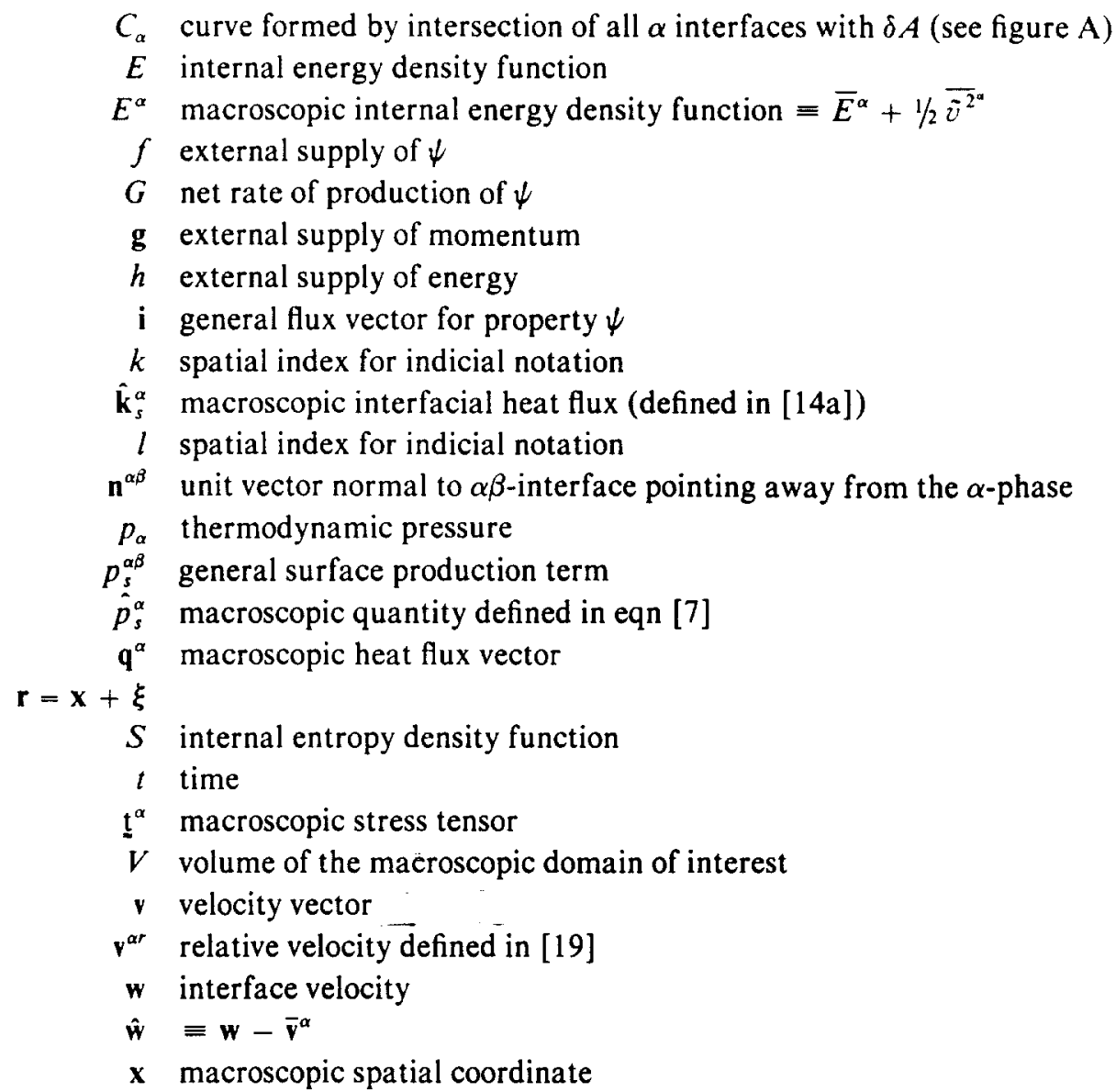

\section{Greek letters}

$\alpha$ refers to $\alpha$ phase

$\beta$ refers to $\beta$ phase

$\Gamma$ entropy production

$\gamma_{\alpha}$ phase distribution function $=\left\{\begin{array}{l}1 \text { in } \alpha \text { phase } \\ 0 \text { not in } \alpha \text { phase }\end{array}\right.$

$\delta A$ infinitesimal element of averaging area

$\delta A_{\alpha \beta}$ area of all $\alpha-\beta$ interfaces within REV

$\delta V$ averaging volume

$\epsilon_{\alpha} \quad$ volume fraction of $\alpha$ th phase

$\eta_{s}^{\alpha}$ surface entropy flux defined by [15]

$\theta$ thermodynamic temperature

$\theta^{\alpha r}$ relative temperature defined in [19]

$\lambda_{s}^{\alpha \beta^{*}}$ general surface flux

$\mu$ surface coordinate index

$\nu_{\alpha \beta}^{\prime}$ surface coordinate unit vector shown in figure A with components $\left(\nu_{\alpha \beta}^{\prime}\right)^{\mu}$

$\xi$ position in space with respect to macroscopic coordinate

$\rho$ mass density

$\sigma_{s}^{\alpha}$ macroscopic surface stress tensor defined by [12] with spatial components $\left(\sigma_{s}^{\alpha}\right)^{i j}$

$\sigma_{s}^{\alpha \beta^{\sigma}} \quad$ microscopic surface stress tensor defined prior to eqn [12]

$\phi$ entropy flux

$\psi$ typical intrinsic thermomechanical property 


\section{REFERENCES}

ARIS, R. 1962 Vectors, Tensors, and the Basic Equations of Fluid Mechanics. PrenticeHall, Englewood Cliffs, NJ.

Bedford, A. \& DRUMHelleR, D. S. 1983 Recent advances, theories of immiscible and structured mixtures. Int. J. Engng Sci. 21, 863-960.

Bowen, R. M. 1982 Porous medial model formulations by the theory of mixtures. Proc. NATO Advanced Study Institute, Mechanics of Fluids in Porous Media, New Approaches in Research, July 18-27, U. of Delaware.

Delhaye, J. M. 1974 Jump conditions and entropy sources in two-phase systems, local instant formulation. Int. J. Multiphase Flow 1, 395-409.

Delhaye, J. M. 1977 Instantaneous space-averaged equations. in Two-Phase Flows and Heat Transfer, Vol. I, (S. Kakac and F. Mayinger, eds.). Hemisphere Publ., Washington, D.C. $81-90$.

Drew, D. A. 1971 Averaged field equations for two-phase media. Studies in Appl. Math. 50, 133-166.

DREw, A. D. \& SEgel, L. A. 1971 Averaged equations for two-phase flows. Studies in Appl. Math. 50, 205-231.

ENE, H. I. 1981 On the thermodynamic theory of mixtures. Int. J. Engng Sci. 19, 905-914.

GraY, W. G. 1975 A derivation of the equations for multi-phase transport. Chem. Engng Sci. 30, 229-233.

GRAY, W. G. 1983 Local volume averaging of multiphase systems using a non-constant averaging volume. Int. J. Multiphase Flow 9, 755-761.

HASSANIZADEH, M. 1979, September Macroscopic description of multi-phase systems-A thermodynamic theory of flow in porous media. Ph.D. Thesis, Princeton University.

HASSANIZADEH, M. \& GRAY, W. G. 1979a General conservation equations for multi-phase systems: 1. Averaging procedure. Adv. in Water Res. 2, 131-144.

HASSANIZADEH, M. \& GRAY, W. G. $1979 \mathrm{~b}$ General conservation equations for multi-phase systems: 2. Mass, momenta, energy and entropy equations. Adv. Water Res. 2, 191-208.

IsHII, M. 1975 Thermo-Fluid Dynamic Theory of Two-Phase Flow. Eyrolles, Paris.

MoECKEl, G. P. 1975 Thermodynamics of an interface. Arch. Rat. Mech. Anal. 57, 255-280.

Nigmatulin, R. I. 1979 Spatial averaging in the mechanics of heterogeneous and dispersed systems. Int. J. Multiphase Flow 5, 353-385.

Passman, S. L., Nunziato, J. W. \& Walsh, E. K. 1983, March A theory of multiphase mixtures. Sandia Report SAND 82-2261.

SCRIVEN, L. E. 1960 Dynamics of a fiuid interface. Chem. Engng Sci. 12, 98-108.

SLATTERY, J. C. 1964 Surfaces-I. momentum and momentum-of-momentum balances for moving surfaces. Chem. Engng Sci. 19, 379-385.

Slattery, J. C. 1967 General balance equation for a phase interface. Ind. Eng. Chem. Fund. 6, 108-115.

\section{APPENDIX A}

Application of Green's Theorem to Interfacial Flux Term

Green's theorem in a surface may be stated as (Aris 1962):

$$
\int_{S} \nabla^{\prime} \cdot \mathbf{f}^{\prime} \mathrm{d} a=\oint_{C} \boldsymbol{\nu}^{\prime} \cdot \mathbf{f}^{\prime} \mathrm{d} l
$$

for a given surface $S$ and its bounding curve $C$. Here $\nu^{\prime}$ is a surface coordinate unit vector lying in $S$ and normal to $C$. A corollary of [A.1] is that for any closed surface, $\hat{S}$ :

$$
\int_{\hat{S}} \nabla^{\prime} \cdot \mathbf{f}^{\prime} \mathrm{d} a=0 .
$$


Identity [A.2] holds as long as $\mathbf{f}^{\prime}$ is continuous across any curve in $\hat{S}$. This corollary may be easily derived from [A.1] by splitting the surface $\hat{S}$ into two parts with some curve $\hat{C}$ and then summing the resultant integrals.

Now consider a surface integral over all $\alpha-\beta$ interfaces $\left(A_{\alpha \beta}\right)$ in a volume $V$ (see figure A]:

$$
\int_{A_{\alpha \beta}} \nabla^{\prime} \cdot \lambda_{s}^{\alpha \beta^{\prime}} \mathrm{d} a
$$

If the volume $V$ is divided into $m$ subvolumes $\left(\delta V_{i}\right)$ [A.3] can be expanded as a sum of integrals over the interfacial areas in each subregion $\left(\delta A_{\alpha \beta_{1}}\right)$ :

$$
\int_{A_{\alpha \beta}} \nabla^{\prime} \cdot \lambda_{s}^{\alpha \beta^{\prime}} \mathrm{d} a=\sum_{i=1}^{m}\left\{\sum_{\alpha} \sum_{\beta \neq \alpha} \int_{\delta A_{\alpha \alpha_{i}}} \nabla^{\prime} \cdot \lambda_{s}^{\alpha \beta^{\prime}} \mathrm{d} a\right\} .
$$

The aforementioned division into subvolumes is arbitrary so that if $M$ different divisions are considered [A.4] could be rewritten as:

$$
\int_{A_{\alpha \beta}} \nabla^{\prime} \cdot \lambda_{s}^{\alpha \beta^{\prime}} \mathrm{d} a=\sum_{j=1}^{M} \sum_{i=1}^{m_{j}} \frac{1}{\delta V_{i j}}\left[\sum_{\alpha} \sum_{\beta \neq \alpha} \int_{\delta A_{\alpha \beta_{i j}}} \nabla^{\prime} \cdot \lambda_{s}^{\alpha \beta^{\prime}} \mathrm{d} a\right] \frac{\delta V_{i j}}{M} .
$$

Here $\delta V_{i j}$ is the $i$ th subvolume-in the $j$ th set. Since [A.5] is summed over all sets, division by $M$ is necessary to preserve the equality. If the requirement is imposed that no two subvolumes have the same centroid (Hassanizadeh \& Gray (1979a)), [A.5] becomes in the limit as $M \rightarrow \infty$ :

$$
\int_{A_{\alpha s}} \nabla^{\prime} \cdot \lambda_{s}^{\alpha \beta^{\prime}} \mathrm{d} a=\int_{V} \sum_{\alpha} \sum_{\beta \neq \alpha} \frac{1}{\delta V} \int_{\delta A_{\alpha s}} \nabla^{\prime} \cdot \lambda_{s}^{\alpha \beta^{\prime}} \mathrm{d} a \mathrm{~d} V
$$

In view of corollary [A.2], only those interfacial areas which do not form closed surfaces within $V$ will contribute to the integral [A.3]. The only surfaces $\left(\delta A_{\alpha \beta}\right)$ which are not closed in $V$ are those which intersect $A$, the boundary of $V$. Let $A$ be subdivided into $m$ subareas $\left(\delta A_{i}\right)$. Then, by the use of Green's theorem [A.1], integral [A.3] can be expanded as:

$$
\int_{A_{\alpha \beta}} \nabla^{\prime} \cdot \lambda_{s}^{\alpha \beta^{\prime}} \mathrm{d} a=\sum_{i=1}^{m}\left\{\sum_{\alpha} \int_{C_{e^{\prime}}} \nu_{\alpha \beta}^{\prime} \cdot \lambda_{s}^{\alpha \beta^{\prime}} \mathrm{d} l\right\}
$$

Here $C_{\alpha_{i}}$ is the curve formed by the intersection of all interfaces which contact that $\alpha$ phase with the subarea $\delta A_{i}$ and $\nu_{\alpha \beta}^{\prime}$ is a two-dimensional unit vector lying inside the $\alpha-\beta$ interface and normal to $C_{\alpha_{i}}$ As in the development of [A.5], different subdivisions of $A$ are possible and [A.7] can be rewritten:

$$
\int_{A_{\infty}} \nabla^{\prime} \cdot \lambda_{s}^{\alpha \beta^{\prime}} \mathrm{d} a=\sum_{j=1}^{M} \sum_{i=1}^{m_{j}} \frac{1}{\delta A_{i j}}\left[\sum_{\alpha} \int_{C_{a i j}} \nu_{\alpha \beta}^{\prime} \cdot \lambda_{s}^{\alpha \beta^{\prime}} \mathrm{d} l\right] \frac{\delta A_{i j}}{M} .
$$

Taking the limit of [A.8] as $M \rightarrow \infty$ yields:

$$
\int_{A_{\alpha \beta}} \nabla^{\prime} \cdot \lambda_{s}^{\alpha \beta^{\prime}} \mathrm{d} a=\int_{A} \sum_{\alpha} \frac{1}{\delta A} \int_{C_{\alpha}} \nu_{\alpha \beta}^{\prime} \cdot \lambda_{s}^{\alpha \beta^{\prime}} \mathrm{d} l \mathrm{~d} A
$$


Comparison of [A.6] and [A.9] produces the final result:

$$
\int_{V} \sum_{\alpha} \sum_{\beta \neq \alpha} \frac{1}{\delta V} \int_{\delta A_{\alpha s}} \nabla^{\prime} \cdot \lambda_{s}^{\alpha \beta^{\prime}} \mathrm{d} a \mathrm{~d} V=\int_{A} \sum_{\alpha} \frac{1}{\delta A} \int_{C_{*}} \nu_{\alpha \beta}^{\prime} \cdot \lambda_{s}^{\alpha \beta^{\prime}} \mathrm{d} l \mathrm{~d} A
$$

It should be noted that the averaged integrals over $\delta A_{\alpha \beta}$ and $C_{\alpha}$ which appear in equation [A.10] are dependent upon the characteristic length of the representative elementary volume and area but not upon the shape of the elements.

\section{APPENDIX B}

\section{Examination of the Symmetry of $\sigma_{s}^{\alpha}$}

In this appendix, the linear and angular momentum jump conditions are manipulated to gain information about the interfacial stress tensor, $\sigma_{s}^{\alpha}$. The following development is a condensation of a more complete discussion of this subject which appears in Hassanizadeh (1979).

With reference to table 1 , the interface constraint [9] for angular momentum may be written as:

$$
\begin{aligned}
\sum_{\alpha}\left\{\frac{1}{\delta V} \sum_{\beta \neq \alpha} \int_{\delta A_{\alpha \beta}} \rho(\mathbf{r} \times \mathbf{v}) \cdot(\mathbf{w}-\mathbf{v}) \cdot \mathbf{n}^{\alpha \beta} \leq \mathrm{d} a\right. & \left.+\frac{1}{\delta V} \sum_{\beta \neq \alpha} \int_{\delta A_{\alpha \beta}} \mathbf{n}^{\alpha \beta} \cdot(\mathbf{r} \times \mathrm{t}) \mathrm{d} a\right\} \\
& =\frac{1}{2} \sum_{\alpha} \nabla \cdot\left[\frac{1}{\delta A} \int_{C_{\alpha}} \nu_{\alpha \beta}^{\prime} \cdot\left(\mathbf{r} \times \boldsymbol{\sigma}_{s}^{\alpha \beta^{\prime}}\right) \mathrm{d} l\right] .
\end{aligned}
$$

Recalling that $\mathbf{r}=\mathbf{x}+\boldsymbol{\xi},[\mathrm{B} .1]$ can be rearranged to yield:

$$
\begin{aligned}
\sum_{\alpha}\left\{\mathbf{x} \times\left[\langle\rho\rangle_{\alpha} e^{\alpha}(\rho \overline{\mathbf{v}})\right.\right. & \left.+\langle\rho\rangle_{\alpha} e^{\alpha}(\rho) \overline{\mathbf{v}}^{\alpha}+\langle\rho\rangle_{\alpha} \hat{\mathbf{T}}^{\alpha}\right] \\
& \left.+\langle\rho\rangle_{\alpha}\left[e^{\alpha}(\rho \xi \times \mathbf{v})+{ }_{\alpha} \hat{\mathbf{M}}^{\alpha}\right]\right\}=\frac{1}{2} \sum_{\alpha} \nabla \cdot\left(\mathbf{x} \times g_{s}^{\alpha}\right)+\nabla \cdot{ }_{a} \underline{m}_{s}^{\alpha},
\end{aligned}
$$

where

$$
{ }_{a} \hat{\mathbf{M}}^{\alpha}=\frac{1}{\langle\rho\rangle_{\alpha} \delta V} \sum_{\beta \neq \alpha} \int_{\delta A_{\alpha S}} \xi \times \underline{t} \cdot \mathbf{n}^{\alpha \beta} \mathrm{d} a \quad \begin{aligned}
& \text { is a supply of angular } \\
& \text { momentum due to mechan- } \\
& \text { ical interactions. }
\end{aligned}
$$

and

$$
\mathrm{N} \cdot{ }_{a} \mathrm{~m}_{s}^{\alpha}={ }_{a} \mathrm{~m}_{s}^{\alpha}=\frac{1}{\delta A} \int_{C_{\alpha}} \nu_{\alpha \beta}^{\prime} \cdot\left(\xi \times q_{s}^{\alpha \beta \prime}\right) \mathrm{d} l \quad \begin{aligned}
& \text { is an apparent macro- } \\
& \text { scopic stress couple } \\
& \text { vector due to interfacial } \\
& \text { stress. }
\end{aligned}
$$

Constraint [B.2] may be further simplified by incorporating the linear momentum constraint [12] and using the identity $\nabla \mathbf{x}=\mathrm{I}$ :

$$
\sum_{\alpha}\langle\rho\rangle_{\alpha}\left[e^{\alpha}(\rho \xi \times \mathbf{v})+{ }_{a} \hat{\mathbf{M}}^{\alpha}\right]=\frac{1}{2} \sum_{\alpha}\left\{\mathrm{I} \times \cdot \sigma_{s}^{\alpha}+\nabla \cdot{ }_{\alpha} \mathrm{m}_{s}^{\alpha}\right\}
$$

A macroscopic interface condition for local spin can also be derived by taking the cross 
852

L. M. ABRIOLA and W. G. GRAY

product of $\boldsymbol{\xi}$ with the microscopic linear momentum jump condition [2]:

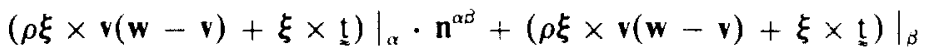

$\cdot \mathbf{n}^{\beta \alpha}=\nabla^{\prime} \cdot\left(\xi \times \sigma_{s}^{\alpha \beta^{\prime}}\right)$,

and then averaging this equation over an REV to achieve:

$$
\sum_{\alpha}\langle\rho\rangle_{\alpha}\left(e^{\alpha}(\rho \xi \times \mathbf{v})+{ }_{a} \hat{\mathbf{M}}^{\alpha}\right)=\frac{1}{2} \sum_{\alpha} \nabla \cdot{ }_{\alpha} \underline{\underline{m}}_{s}{ }^{\alpha} .
$$

Constraint [B.5] is valid for microscopically nonpolar materials. Comparison of [B.3] and [B.5] yields the result

$$
\mathrm{I} \times \cdot \sum_{\alpha} \boldsymbol{g}_{s}^{\alpha}=0
$$

or

$$
\sum_{\alpha} \sigma_{s_{i j}}^{\alpha}=\sum_{\alpha} \sigma_{s_{j i}}^{\alpha}
$$

